\title{
The Effectiveness of ${ }^{18}$ F-FDG PET/CT Combined with STIR MRI for Diagnosing Nodal Involvement in the Thorax
}

Miwa Morikawa ${ }^{1}$, Yoshiki Demura ${ }^{1}$, Takeshi Ishizaki ${ }^{1}$, Shingo Ameshima ${ }^{1}$, Isamu Miyamori ${ }^{2}$, Masato Sasaki ${ }^{3}$, Tatsuro Tsuchida ${ }^{4}$, Hirohiko Kimura ${ }^{4}$, Yasuhisa Fujibayashi ${ }^{5}$, and Hidehiko Okazawa ${ }^{5}$

${ }^{I}$ Department of Respiratory Medicine, University of Fukui, Fukui, Japan; ${ }^{2}$ Third Department of Internal Medicine, University of Fukui, Fukui, Japan; ${ }^{3}$ Department of Thoracic Surgery, University of Fukui, Fukui, Japan; ${ }^{4}$ Department of Radiology, University of Fukui, Fukui, Japan; and ${ }^{5}$ Biomedical Imaging Research Center, University of Fukui, Fukui, Japan

The purpose of this study was to compare the efficacy of short- $\tau$ inversion-recovery (STIR) MRI and ${ }^{18} \mathrm{~F}-\mathrm{FDG} \mathrm{PET} / \mathrm{CT}$ for the detection of metastasis in mediastinal and hilar lymph nodes in patients with lung cancer. Methods: Ninety-three patients with known or suspected lung cancer with mediastinal and hilar lymph node swelling underwent STIR MRI and ${ }^{18} \mathrm{~F}-\mathrm{FDG}$ PET/CT examinations. STIR MRI scans were obtained with a $2 \%$ copper sulfate phantom placed along the back of each patient, with the lymph node-to-phantom ratio calculated for quantitative analysis. For qualitative analysis, the results of all STIR MRI scans were evaluated using a 5-point visual scoring system. To evaluate the diagnostic capabilities of STIR MRI and ${ }^{18} \mathrm{~F}-\mathrm{FDG}$ PET/CT, we used receiver-operating-characteristic curve analysis to determine the optimal thresholds for the lymph node-to-phantom ratio, visual score, and maximal standardized uptake value. Further, the capability of each to determine N-stage was compared in each patient using the McNemar test. Results: A total of 137 lymph nodes (82 malignant lesions, 55 benign lesions) were analyzed. When optimal threshold values were adopted, the quantitative and qualitative sensitivity, specificity, and accuracy of STIR MRI were not significantly different from those of ${ }^{18} \mathrm{~F}-\mathrm{FDG}$ $\mathrm{PET} / \mathrm{CT}$. However, ${ }^{18} \mathrm{~F}-\mathrm{FDG}$ PET/CT in combination with qualitative STIR MRI analysis had a significantly higher capability to detect nodal involvement on an individual-patient basis $(96.9 \%$ specificity, $90.3 \%$ accuracy) than did ${ }^{18} \mathrm{~F}-\mathrm{FDG}$ PET/CT alone (65.6\% specificity, $81.7 \%$ accuracy). Conclusion: We found that the diagnostic capability of STIR MRI was not significantly different from that of ${ }^{18} \mathrm{~F}-\mathrm{FDG}$ PET/CT. However, when those methods were combined, the diagnostic capability for $\mathrm{N}$-staging was significantly improved.

Key Words: lung cancer; ${ }^{18}$ F-FDG PET/CT; STIR MR imaging; $\mathrm{N}$-staging

J Nucl Med 2009; 50:81-87

DOI: 10.2967/jnumed.108.056408

Received Jul. 29, 2008; revision accepted Oct. 8, 2009.

For correspondence or reprints contact: Yoshiki Demura, Department of Respiratory Medicine, University of Fukui, 23-3 Matsuokashimoaizuki,

Eiheiji-cho, Yoshida-gun Fukui 910-1193 Japan.

E-mail: DEM2180@aol.com

COPYRIGHT $\odot 2009$ by the Society of Nuclear Medicine, Inc.
I maging with ${ }^{18}$ F-FDG PET, which is used to detect the biologic activities of tumor cells, has been increasingly applied for diagnosing, staging, and determining the type of lung cancer $(1,2)$. However, recent studies have reported poor accuracy for ${ }^{18} \mathrm{~F}$-FDG PET in terms of lung cancer nodal staging (3-5). According to the evidence-based clinical practice guidelines of the American College of Chest Physicians, it is recommended that further evaluation of the mediastinum with sampling of the abnormal lymph node should be performed before surgical resection of the primary tumor in patients with an abnormal result on ${ }^{18} \mathrm{~F}$-FDG PET scans (6). In addition, several investigators have reported that integrated PET/CT is superior to conventional contrastenhanced CT or ${ }^{18} \mathrm{~F}$-FDG PET alone for detecting nodal metastasis in patients with non-small cell lung cancer $(3,7,8)$. However, PET/CT staging of lymph node involvement does not replace the need for mediastinoscopy $(9,10)$.

Mediastinoscopy is the gold standard for mediastinal lymphatic staging because of its remarkable sensitivity of approximately $80 \%$, with a specificity of, by definition, $100 \%(11,12)$. Nevertheless, mediastinoscopy has the risk of life-threatening complications, such as hemorrhage, mediastinitis, pneumothorax, and vocal cord paresis (13). Thus, it would be preferable to develop less invasive procedures that would also have improved diagnostic capabilities. ${ }^{18}$ F-FDG PET can reflect physiologic and biochemical functions and is useful for differential diagnosis of benign and malignant lesions (14-16). However, there are numerous causes of ${ }^{18} \mathrm{~F}-\mathrm{FDG}$ uptake in benign processes, which lead to false-positive results $(1,17-19)$ even when PET/CT is also used $(20,21)$. Some investigators have reported that short- $\tau$ inversion-recovery (STIR) MRI has utility for the detection of metastases in mediastinal and hilar lymph nodes in lung cancer patients (22-24).

On the basis of these backgrounds, we hypothesized that STIR MRI would improve the accuracy of ${ }^{18}$ F-FDG PET for $\mathrm{N}$-staging in patients with lung cancer. To test our 
hypothesis, we prospectively evaluated and compared the accuracy of STIR MRI, ${ }^{18} \mathrm{~F}-\mathrm{FDG}$ PET/CT, and ${ }^{18} \mathrm{~F}-\mathrm{FDG}$ PET combined with STIR MRI for the diagnosis of nodal involvement in patients suspected to have lung cancer.

\section{MATERIALS AND METHODS}

\section{Patients}

The study was approved by the Institutional Review Board of our hospital, and written informed consent was obtained from all patients who participated. We prospectively studied patients with known or suspected lung cancer and mediastinal and hilar lymph node swelling detected by chest CT. Mediastinal and hilar lymph nodes were assessed if the short-axis diameter on transaxial chest CT images was greater than $10 \mathrm{~mm}$. Patients with hyperglycemia (blood glucose levels $>126 \mathrm{mg} / \mathrm{dL}$ ) at the time of ${ }^{18} \mathrm{~F}-\mathrm{FDG}$ injection were excluded from the study. All patients underwent STIR MRI and ${ }^{18} \mathrm{~F}$-FDG PET/CT before a biopsy and treatment for lung cancer, and the 2 examinations were completed within 1 wk of each other. STIR MRI and ${ }^{18} \mathrm{~F}-\mathrm{FDG}$ PET/CT findings for all primary lesions and nodal involvement were compared with the histopathologic diagnosis within 4 wk. Histopathologic diagnoses of all mediastinal and hilar lymph nodes were confirmed by the results of surgical resection, mediastinoscopy, or transbronchial needle aspiration. Because the negative predictive value of transbronchial needle aspiration is considered to be low (25), surgical confirmation was always obtained in cases that had no malignant lymph node invasion demonstrated by that endoscopic technique.

\section{CT Examination}

CT was performed using a 16-detector-row CT system (Sensation 16; Siemens). Continuous 5-mm-thick sections were obtained at $5-\mathrm{mm}$ intervals from the lung apices to the adrenal glands before and during an intravenous bolus injection of contrast material (iopamidol [Iopamiron 300; Bayer] or iohexol [Omnipaque 300; GE Healthcare]) given at $2 \mathrm{~mL} / \mathrm{s}$ with a power injector. Transaxial images were obtained for all patients.

\section{MRI}

MRI examination was performed using a 1.5-T superconducting magnet (Signa Excite; GE Healthcare) with a body coil. In all patients, axial electrocardiographically triggered STIR fast spinecho MRI scans (repetition time, 4,000-14,285 ms; effective echo time, $58 \mathrm{~ms}$; inversion time, $150 \mathrm{~ms}$; number of excitations, 2; slice thickness, $7 \mathrm{~mm}$; slice gap, $1 \mathrm{~mm}$ ) were obtained with a $2 \%$ copper sulfate phantom (26), which was placed along the back of each patient (23).

\section{${ }^{18}$ F-FDG PET/CT Examination}

All ${ }^{18}$ F-FDG PET/CT examinations were performed with a whole-body scanner (Discovery LS; GE Healthcare). All patients fasted overnight (for at least $12 \mathrm{~h}$ ) before radiotracer administration. ${ }^{18} \mathrm{~F}$-FDG PET images from the skull through the mid thigh were obtained $50 \mathrm{~min}$ after intravenous injection of ${ }^{18} \mathrm{~F}-\mathrm{FDG}$ at a dose $(185 \mathrm{MBq})$ that is commonly used for clinical ${ }^{18} \mathrm{~F}$-FDG PET examinations in Japan, and CT-based attenuation correction was performed. CT-based attenuation correction factors were then applied to the emission data, and the attenuation-corrected emission images were reconstructed using an ordered-subset expectation maximization iterative reconstruction algorithm (2 iterations and 14 subsets). The reconstructed images were converted to standardized-uptake-value (SUV) images using patient body weight and dose of ${ }^{18} \mathrm{~F}-\mathrm{FDG}$ (tumor activity concentration/injected dose/ body weight).

\section{STIR MRI Scan Analysis}

On axial images obtained by STIR MRI, signal intensity (SI) was measured in a circular region of interest drawn over each lymph node diagnosed as malignant on CT to determine its size and the phantom of $2 \%$ copper sulfate. Each region of interest drawn over the lymph node encompassed the entire cross-sectional area of the node and the phantom (10 $\mathrm{mm}$ in diameter). To quantitatively evaluate the SI of each of those lymph nodes, we calculated the lymph node-to-phantom ratio (LPR) using the following formula (23):

\section{LPR $=$ average SI of lymph node/average SI of phantom}

To determine the ability to perform qualitative analysis using STIR MRI scans, the results of all examinations were prospectively evaluated by 2 experienced radiologists using a 5-point visual scoring system according to the method of Ohno et al. (23), as follows: 1, SI of lymph node could not be evaluated; 2, SI of lymph node was less than or equal to SI of mediastinal fat; 3 , SI of lymph node was greater than SI of mediastinal fat and less than or equal to SI of muscle; 4, SI of lymph node was greater than SI of muscle and less than or equal to SI of primary lesion; and 5, SI of lymph node was greater than SI of primary lesion. The final diagnosis of lymph node metastasis was made after the 2 reviewers reached a consensus.

\section{Analysis of ${ }^{18} \mathrm{~F}-\mathrm{FDG}$ PET/CT Images}

${ }^{18} \mathrm{~F}$-FDG PET images were interpreted independently and prospectively by an experienced radiologist and nuclear medicine physician, without knowledge of histopathologic or other radiologic data. Semiquantitative analysis of the ${ }^{18} \mathrm{~F}-\mathrm{FDG}$ uptake was based on region-of-interest analysis that produced maximal SUV and mean SUV. Swollen lymph nodes were evaluated using ${ }^{18} \mathrm{~F}-\mathrm{FDG}$ PET/CT. A region of interest was drawn over each mediastinal or hilar lymph node at the most active site on ${ }^{18} \mathrm{~F}-\mathrm{FDG}$ PET/CT images by the same radiologist and experienced nuclear medicine physician.

\section{Statistical Analysis}

To determine observer performance for qualitatively analyzed STIR MRI scans on a per-node basis, a $\kappa$-statistic was used. Interobserver agreement was considered as slight for a $\kappa$ of less than 0.21 , fair for a $\kappa$ of $0.21-0.40$, moderate for a $\kappa$ of $0.41-0.60$, substantial for a $\kappa$ of $0.61-0.80$, and nearly perfect for a $\kappa$ of $0.81-$ 1.00 (27). To evaluate the ability of the LPR and visual score to enable differentiation of lymph nodes with malignancy from benign nodes, we used the Student $t$ test. The Student $t$ test was also used to evaluate the diagnostic capability of STIR MRI and ${ }^{18} \mathrm{~F}-\mathrm{FDG}$ PET/CT while the optimal thresholds of LPR, visual score, maximal SUV, and mean SUV were determined using receiver-operating-characteristic curve-based analysis. The sensitivity, specificity, and diagnostic accuracy of each modality were determined. The diagnostic accuracy of each method was compared using a McNemar test. For all statistical analyses, $P$ values of less than 0.05 were considered to be statistically significant. 


\section{RESULTS}

Subject Demography and Clinical Characteristics

We studied 93 consecutive patients evaluated between January 2005 and June 2008 (age range, 30-87 y; mean age $\pm \mathrm{SD}, 66.1 \pm 10.9 \mathrm{y} ; 76$ men and 17 women) (Table 1$)$ and evaluated 137 lymph nodes, of which 82 were pathologically diagnosed as malignant and 55 as benign (Table 2). In the group without malignancy, 11 cases of sarcoidosis were included. For those 11 patients, the diagnosis of sarcoidosis was based on compatible clinical and follow-up findings, histologic evidence of noncaseating epithelioid cell granulomas, and exclusion of known causes of granulomatous diseases according to the consensus statement by the American Thoracic Society, European Respiratory Society, and World Association of Sarcoidosis and Other Granulomatous Disorders (28). The lymph nodes ranged in size from 10 to $36 \mathrm{~mm}$, and the mean size of nodes with malignancy was significantly greater than that of benign nodes (mean, $18.4 \pm$ $6.2 \mathrm{~mm}$ vs. $13.9 \pm 3.1 \mathrm{~mm}, P<0.0001$, analyzed by Student $t$ test).

\section{LPR and Visual Scores for Malignant and Benign Lymph Nodes}

The mean LPR values for malignant and benign lymph nodes were $0.22 \pm 0.32$ and $0.08 \pm 0.03$, respectively $(P<$ 0.001 , analyzed by Student $t$ test), which was a statistically significant difference. Table 3 shows the results of the rated visual scores based on STIR MRI by the 2 reviewers.

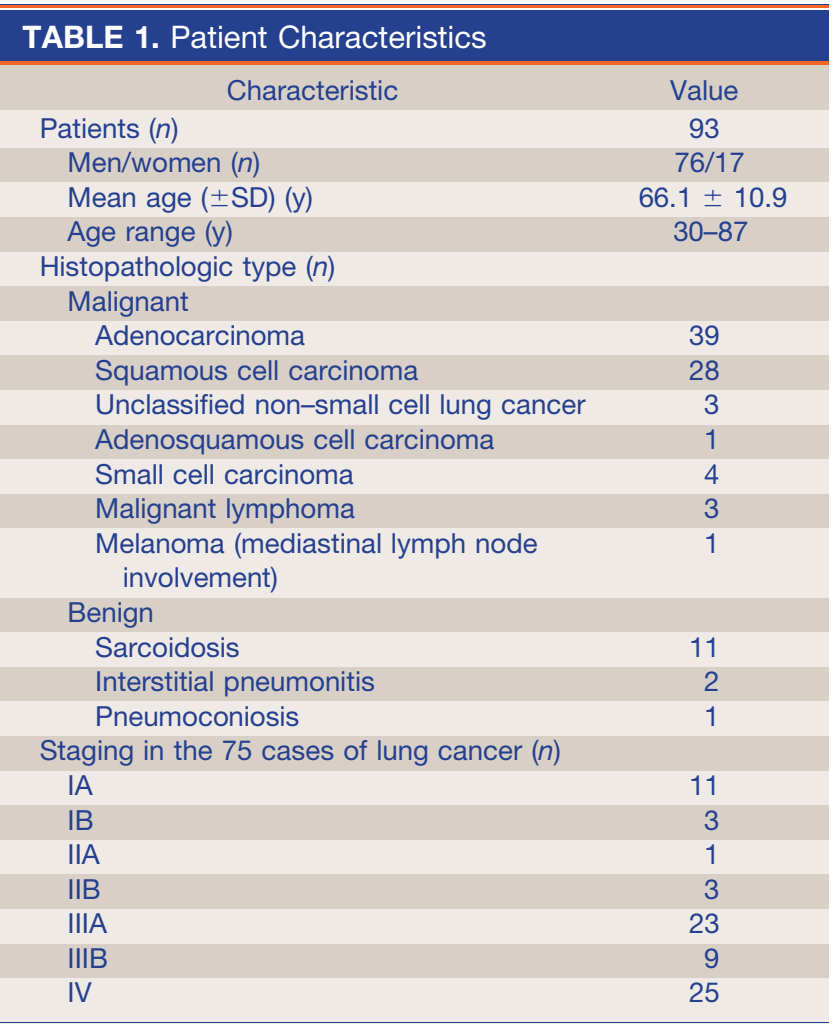

\begin{tabular}{|c|c|}
\hline Characteristic & Value \\
\hline \multicolumn{2}{|l|}{ Location $(n)$} \\
\hline Mediastinum (malignant/benign) & $118(71 / 47)$ \\
\hline Hilum (malignant/benign) & $19(11 / 8)$ \\
\hline \multicolumn{2}{|l|}{ Mean size $( \pm \mathrm{SD})(\mathrm{mm})$} \\
\hline Malignant & $18.4 \pm 6.2$ \\
\hline Benign & $13.9 \pm 3.1$ \\
\hline \multicolumn{2}{|l|}{ Histopathologic type $(n)$} \\
\hline \multicolumn{2}{|l|}{ Malignant } \\
\hline Adenocarcinoma & 35 \\
\hline Squamous cell carcinoma & 31 \\
\hline Unclassified non-small cell lung cancer & 2 \\
\hline Adenosquamous cell carcinoma & 1 \\
\hline Small cell carcinoma & 6 \\
\hline Malignant lymphoma & 6 \\
\hline Melanoma & 1 \\
\hline \multicolumn{2}{|l|}{ Benign $(n)$} \\
\hline Granulomatous inflammation & 26 \\
\hline Anthracosis & 23 \\
\hline Follicular hyperplasia & 2 \\
\hline Follicular hyperplasia and anthracosis & 1 \\
\hline Nonspecific inflammation & 3 \\
\hline \multicolumn{2}{|l|}{ Method of diagnosis $(n)$} \\
\hline $\begin{array}{l}\text { Surgery or mediastinoscopy } \\
\text { (malignant/benign) }\end{array}$ & $56(19 / 37)$ \\
\hline $\begin{array}{l}\text { Transbronchial needle aspiration } \\
\text { (malignant/benign) }\end{array}$ & $81(63 / 18)$ \\
\hline
\end{tabular}

Interobserver agreement for qualitative analysis of STIR MRI data was substantial $(\kappa=0.76)$.

\section{Accuracy of STIR MRI and ${ }^{18}$ F-FDG PET/CT for Diagnosing Lymph Nodes}

An LPR of 0.1 for quantitative STIR MRI analysis and a visual score of 4 for qualitative analysis were adopted as optimal cutoff values for receiver-operating-characteristic analysis. Receiver-operating-characteristic analysis was also used to evaluate the effectiveness of SUV as a marker for malignant lymph nodes versus benign nodes. Sensitivity, specificity, positive predictive value, negative predictive value, and accuracy were calculated for each level of SUV,

\begin{tabular}{|c|c|c|}
\hline Rated score & Observer 1 & Observer 2 \\
\hline 1 & 0 & 0 \\
\hline 2 & 5 & 3 \\
\hline 3 & 49 & 53 \\
\hline 4 & 70 & 68 \\
\hline 5 & 13 & 13 \\
\hline
\end{tabular}


by varying the level that signified an optimal threshold value (29). A maximal SUV of 4.1 and mean SUV of 3.5 were adopted as optimal cutoff values for analysis. Table 4 shows the optimal cutoff values, sensitivities, specificities, positive and negative predictive values, and accuracies of STIR MRI and ${ }^{18} \mathrm{~F}$-FDG PET/CT for diagnosing mediastinal and hilar lymph nodes. When the optimal cutoff values of LPR, visual score, and maximal SUV were adopted, sensitivity, specificity, and accuracy did not significantly differ between the 2 methods (STIR quantitative analysis vs. ${ }^{18} \mathrm{~F}-\mathrm{FDG}$ PET/CT: $P=0.28$; STIR qualitative analysis vs. ${ }^{18} \mathrm{~F}$-FDG PET/CT: $P=0.35$, analyzed by McNemar test). On the basis of STIR quantitative analysis, 5 lymph nodes were suggested to be artifacts, 3 of which were related to anthracosis and 2 to sarcoidosis. All showed false-positive results. Each node was less than $14 \mathrm{~mm}$ in size, and the mean size of these 5 was $12.0 \pm 2.0 \mathrm{~mm}$.

For cases in which lymph nodes were found positive both on ${ }^{18} \mathrm{~F}-\mathrm{FDG}$ PET/CT and through qualitative analysis of STIR MRI and were determined to be malignant, the results of comparisons of diagnostic capability based on the definition and using only ${ }^{18} \mathrm{~F}-\mathrm{FDG}$ PET/CT are shown in Table 4. According to the criteria noted above, specificity (94.5\%) and accuracy (89.8\%) were significantly higher than those of ${ }^{18}$ F-FDG PET/CT alone $(65.5 \%$ and $80.3 \%$, respectively) ( $P=0.003$, analyzed by McNemar test). A comparison of ${ }^{18} \mathrm{~F}-\mathrm{FDG}$ PET/CT and ${ }^{18} \mathrm{~F}-\mathrm{FDG}$ PET/CT with STIR MRI on an individual-patient basis for the evaluation of nodal staging is shown in Table 5. The accuracy of ${ }^{18}$ F-FDG PET/CT with STIR MRI was significantly higher than that of ${ }^{18} \mathrm{~F}-\mathrm{FDG}$ PET/CT alone $(90.3 \%$ vs. $81.7 \%, P=0.021$, analyzed by McNemar test). In a comparison of ${ }^{18}$ F-FDG PET/CT combined with STIR MRI and STIR MRI alone, the accuracy of the former method was significantly higher on an individual-node basis $(89.8 \%$ vs. $84.7 \%, P=0.035$, analyzed by McNemar test) and individual-patient basis $(90.3 \%$ vs. $81.7 \%, P=0.045$, analyzed by McNemar test). We also evaluated using mean SUV with an optimal cutoff value of 3.5 and obtained similar results. In a comparison of ${ }^{18} \mathrm{~F}-\mathrm{FDG}$ PET/CT combined with STIR MRI and ${ }^{18}$ F-FDG PET/CT using mean SUV alone, the accuracy of the former method was significantly higher on an individual-patient basis $(90.3 \%$ vs. $81.7 \%, P=0.021$, analyzed by McNemar test). When an SUV of less than 2.5 was used as the threshold level for normalcy, as defined by the American College of Chest Physicians, the sensitivity, specificity, and accuracy of ${ }^{18}$ F-FDG PET/CT were $98.4 \%, 37.5 \%$ and $77.4 \%$, respectively, on an individual-patient basis. Adding qualitative STIR MRI analysis to PET/CT also improved specificity and accuracy, to $78.1 \%$ and $87.1 \%$, respectively $(P=$ 0.029 , analyzed by McNemar test).

Figure 1 shows a representative case of adenocarcinoma and mediastinal lymph node metastasis. The results of STIR MRI and ${ }^{18}$ F-FDG PET/CT were both true-positive. The SI of the lymph node in STIR MRI was greater than that of muscle and equal to the primary tumor in the right upper lobe. Figure 2 shows a representative case of malignant lymphoma that also had a true-positive result in both STIR and ${ }^{18}$ F-FDG PET/CT. Figure 3 shows a representative case of sarcoidosis for which only STIR was useful for diagnosis. ${ }^{18}$ F-FDG PET accumulation was intense in the lymph node.

\section{DISCUSSION}

To our knowledge, this is the first report of the utility of ${ }^{18}$ F-FDG PET/CT combined with STIR MRI for the diagnosis of nodal involvement in thoracic malignancy. In the present study, we assessed STIR MRI for the detection of malignant findings in lymph nodes and compared the results with those obtained on ${ }^{18} \mathrm{~F}-\mathrm{FDG}$ PET/CT. Our results clearly suggest an excellent diagnostic ability for those 2 methods used in combination and indicate the possibility that invasive examinations can be eliminated.

For patients with lung cancer, accurate tumor staging is a prerequisite for determining the disease course, because knowing the stage guides the selection of available treatment modalities and predicts survival. Although size criteria using $\mathrm{CT}$ have been applied in conventional assessments of lymph nodes for metastasis, CT has a limited ability to differentiate between benign and malignant lymph nodes.

TABLE 4. Comparisons of Quantitative and Qualitative Diagnostic Capabilities of STIR MRI, ${ }^{18} \mathrm{~F}-\mathrm{FDG}$ PET/CT, and ${ }^{18} \mathrm{~F}-\mathrm{FDG}$ PET/CT with STIR MRI on Individual-Node Basis

$\begin{array}{ccccccc}\text { Modality } & \begin{array}{c}\text { Cutoff } \\ \text { value }\end{array} & \begin{array}{c}\text { Sensitivity } \\ (\%)\end{array} & \begin{array}{c}\text { Specificity } \\ (\%)\end{array} & \text { PPV (\%) } & \text { NPV (\%) } & \text { Accuracy (\%) } \\ \text { STIR (quantitative analysis) } & 0.1 & 96.3(79 / 82) & 67.3(37 / 55) & 81.4(79 / 97) & 92.5(37 / 40) & 84.7(116 / 137) \\ \text { STIR (qualitative analysis) } & 4 & 93.9(77 / 82) & 70.9(39 / 55) & 82.8(77 / 93) & 88.6(39 / 44) & 84.7(116 / 137) \\ \text { PET/CT (maximal SUV) } & 4.1 & 90.2(74 / 82) & 65.5(36 / 55) & 79.6(74 / 93) & 81.8(36 / 44) & 80.3(110 / 137) \\ \text { PET/CT(maximal SUV) + STIR } & - & 86.6(71 / 82) & 94.5^{*}(52 / 55) & 95.9(71 / 74) & 82.5(52 / 63) & 89.8^{*}(123 / 137) \\ \text { (qualitative analysis) } & & & & & & \end{array}$

*Significant difference compared with ${ }^{18} \mathrm{~F}-\mathrm{FDG} \mathrm{PET} / \mathrm{CT}$ using McNemar test $(\mathrm{P}<0.05)$.

PPV $=$ positive predictive value; NPV = negative predictive value.

Sensitivity, specificity, and accuracy were not significantly different between the 2 methods. 


\begin{tabular}{|c|c|c|c|c|c|c|}
\hline Modality & Cutoff value & Sensitivity (\%) & Specificity (\%) & PPV (\%) & NPV (\%) & Accuracy (\%) \\
\hline PET/CT & 4.1 & $90.2(55 / 61)$ & $65.6(21 / 32)$ & $83.3(55 / 66)$ & $77.8(21 / 27)$ & $81.7(76 / 93)$ \\
\hline PET/CT + STIR (qualitative analysis) & - & $86.9(53 / 61)$ & $96.9 *(31 / 32)$ & $98.1(53 / 54)$ & $79.5(31 / 39)$ & $90.3^{*}(84 / 93)$ \\
\hline
\end{tabular}

Recent studies have shown both advantages and limitations of ${ }^{18}$ F-FDG PET/CT for nodal staging and cast doubt on its specificity $(3-5,10,19-21)$. Ohno et al. reported that STIR MRI is more accurate and should be considered as valuable as ${ }^{18} \mathrm{~F}$-FDG PET/CT for the assessment of N-stage of patients with non-small cell lung cancer (22). Previous studies that evaluated the results and utility of STIR MRI for diagnosing mediastinal nodal involvement in lung cancer patients have reported sensitivity of $86 \%-100 \%$, specificity of $86 \%-97 \%$, and accuracy of $86 \%-96 \%(22-24)$.

In the present study, the specificities of STIR MRI and ${ }^{18}$ F-FDG PET/CT were lower than in previous studies. STIR enhances differences between the water content of various tissues, as increasing the $\mathrm{T} 1$ or $\mathrm{T} 2$ value in the tissue increases the relative SI in this imaging modality. Thus, false-positive cases are increased because not only the malignant tumor but also inflammation or lymphatic edema prolongs T1 and T2 relaxation times (30). Moreover, respiratory motion can result in prominent artifacts $(31,32)$. False-positive results obtained by STIR in the present patients were cases of inflammation, such as active sarcoidosis, which increases the serum angiotensin-converting enzyme level, and motion artifacts. In the present study, electrocardiographically triggered STIR was used; thus, artifacts of cardiac motion could be reduced while noise

A
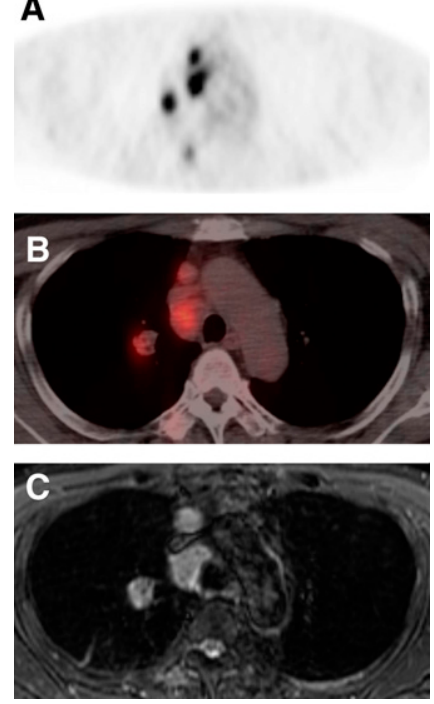

FIGURE 1. A 60 -y-old man with lung adenocarcinoma and metastasis in mediastinal lymph node 3 in pretracheal area. ${ }^{18} \mathrm{~F}-$ FDG PET (A) and integrated ${ }^{18} \mathrm{~F}-\mathrm{FDG} \mathrm{PET} / \mathrm{CT}$ (B) show strong accumulation in primary tumor in right upper lobe and mediastinal lymph nodes (maximal SUV of 11.4 in lymph node 3), and STIR MRI (C) shows lymph node 3 and primary tumor as high-signal-intensity areas. LPR of this lymph node was 0.33 , and rated visual score was 4 . caused by respiratory motion remained. The influence of artifacts mainly increases SI from small lymph nodes, whereas artifacts also cause an increase in false-positive results from STIR quantitative analysis. If a trigger for both cardiac pulse (electrocardiograph) and respiratory motion were possible, the artifacts would be reduced. A pneumatic belt or a compressive cushion placed at the upper abdomen or the chest of the patient was reported as a straightforward method to compensate for respiratory motion (32) and might be useful for this purpose. However, ${ }^{18} \mathrm{~F}-\mathrm{FDG}$ PET/ CT combined with STIR MRI provided a significantly more accurate diagnosis than did STIR MRI alone.

In our observation, false-positive results obtained by ${ }^{18}$ F-FDG PET/CT were cases of sarcoidosis and follicular hyperplasia in lymph nodes caused by obstructive pneumonia. An increase in glucose metabolism by chronic inflammation is the cause of false-positive uptake of ${ }^{18}$ F-FDG PET. In the present results, the specificity of PET/CT was low, at $65.6 \%$ on an individual-patient basis, because a few cases of sarcoidosis determined to be falsepositive were the result of including cases with lymphadenopathy in this prospective study. The causes of false-positive results in STIR (water content) are different from those in ${ }^{18}$ F-FDG PET/CT (increase in glucose metabolism). In patients with sarcoidosis, the lymph nodes become enlarged by
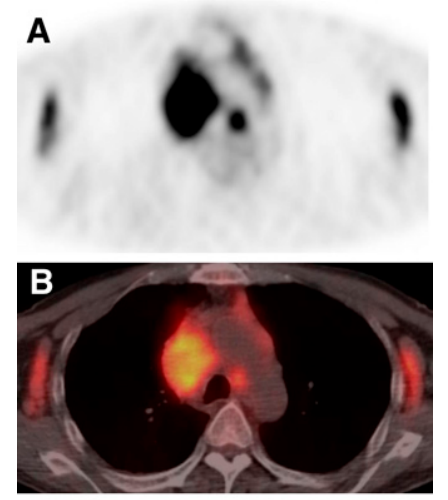

FIGURE 2. A 63-y-old man with malignant lymphoma. ${ }^{18} \mathrm{~F}$-FDG PET (A) and integrated ${ }^{18} \mathrm{~F}-\mathrm{FDG}$ $\mathrm{PET} / \mathrm{CT}$ (B) show strong accumulation in pretracheal and bilateral axillary lymph nodes (maximal SUVs of 10.9-

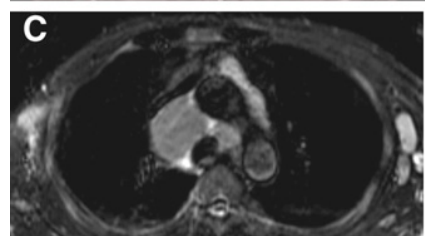
13.6), and STIR MRI (C) shows lymph nodes as high-signal-intensity areas. LPRs were 0.11, and rated visual score was 4 for each node. 
A

FIGURE 3. A 36-y-old man with sarcoidosis. 18F-FDG PET (A) and integrated ${ }^{18} \mathrm{~F}-\mathrm{FDG}$ PET/ CT (B) show strong accumulation in pretracheal and paraaortic lymph nodes (maximal SUVs of 5.3-6.0), and STIR MRI (C) shows lymph nodes as low-signal-intensity areas. LPRs were 0.06, and rated visual score was 2 or 3 for each node.
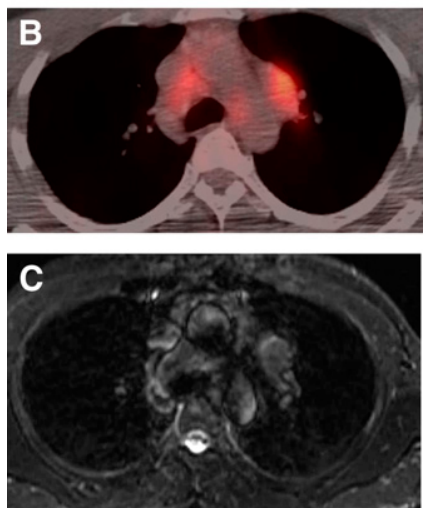

\section{CONCLUSION}

In this study, we compared the diagnostic capability of STIR MRI with that of ${ }^{18} \mathrm{~F}-\mathrm{FDG}$ PET/CT. STIR MRI was able to differentiate false-positive results of ${ }^{18} \mathrm{~F}-\mathrm{FDG}$ PET/ $\mathrm{CT}$ and in combination significantly improved the accuracy of ${ }^{18} \mathrm{~F}$-FDG PET/CT alone. For noninvasive diagnosis of nodal involvement in thoracic malignancy, including lung cancer, STIR MRI for the cases shown positive by ${ }^{18} \mathrm{~F}-\mathrm{FDG}$ $\mathrm{PET} / \mathrm{CT}$ is useful.

\section{ACKNOWLEDGMENTS}

We thank Yasutaka Kawamura, Kameda Medical Center, for his clinical support and helpful comments. This work was supported by the 21st Century COE program "Biomedical Imaging Technology Integration Program," funded by the Japan Society for the Promotion of Science (JSPS). granulomatous formation through the hyperplasia of fibroblasts (33). Therefore, when STIR showed a low intensity, indicating relatively little water content, a differential diagnosis between malignancy and sarcoidosis was obtained. According to our criteria of malignancy diagnosed as positive on both ${ }^{18} \mathrm{~F}$-FDG PET/CT and STIR MRI, there were only 3 false-positive results, 2 nodes related to sarcoidosis and 1 related to follicular hyperplasia, in which the size of all nodes was less than $14 \mathrm{~mm}$. In addition, 11 false-negative nodes were also less than $14 \mathrm{~mm}$ in size. We considered that the spatial resolution of ${ }^{18} \mathrm{~F}-\mathrm{FDG}$ PET/CT and STIR MRI might have been the cause of the false results. With lymph nodes greater than $15 \mathrm{~mm}$, sensitivity, specificity, and accuracy were each $100 \%$.

Previous studies have reported the utility of ${ }^{18} \mathrm{~F}$-FDG PET/CT for mediastinal and extrathoracic staging of nonsmall cell lung cancer $(8,20,34)$. Among the malignant cases in the present study, we also assessed cases of small cell lung cancer, malignant lymphoma, and melanoma. Although the evidence is insufficient for these diseases, the utility of ${ }^{18} \mathrm{~F}$-FDG PET/CT for staging diseases was previously reviewed (35). Thus, it is recommended that ${ }^{18}$ F-FDG PET should be used for diagnosis of various tumors. Moreover, the utility of MRI combined with ${ }^{18} \mathrm{~F}-\mathrm{FDG}$ PET for the assessment of uterine tumors was reported (36). Some investigators have discussed the possibility and utility of PET/MRI $(37,38)$. Because clinical use of PET/STIR MRI results in both anatomic and characteristic images, fine diagnostic capability is expected of this modality. For the assessment of lymph node involvement in thoracic malignancy, including lung cancer, our findings demonstrated the utility of ${ }^{18} \mathrm{~F}$-FDG PET/CT combined with STIR MRI and imply that additional invasive examinations can be eliminated, thereby lowering medical costs and avoiding hazardous diagnostic methods.

\section{REFERENCES}

1. Bunyaviroch T, Coleman RE. PET evaluation of lung cancer. $J$ Nucl Med. 2006;47:451-469.

2. Higashi K, Matsunari I, Ueda Y, et al. Value of whole-body FDG PET in management of lung cancer. Ann Nucl Med. 2003;17:1-14

3. Lardinois D, Weder W, Hany TF, et al. Staging of non-small-cell lung cancer with integrated positron-emission tomography and computed tomography. N Engl J Med. 2003;348:2500-2507.

4. Kernstine KH, Mclaughlin KA, Menda Y, et al. Can FDG-PET reduce the need for mediastinoscopy in potentially resectable nonsmall cell lung cancer? Ann Thorac Surg. 2002;73:394-402.

5. Poncelet AJ, Lonneux M, Coche E, et al. Groupe d'Oncologie Thoracique des Cliniques Saint-Luc. PET-FDG scan enhances but does not replace preoperative surgical staging in non-small cell lung carcinoma. Eur J Cardiothorac Surg. 2001;20:468-474.

6. Alberts WM. Diagnosis and management of lung cancer executive summary: ACCP evidence-based clinical practice guidelines (2nd ed.). Chest. 2007;132: 1S-19S.

7. Reed CE, Harpole DH, Posther KE, et al. Results of the American College of Surgeons oncology group Z0050 trial: the utility of positron emission tomography in staging potentially operable non-small cell lung cancer. $J$ Thorac Cardiovasc Surg. 2003;126:1943-1951.

8. Cerfolio RJ, Ojha B, Bryant AS, Raghuveer V, Mountz JM, Bartolucci AA. The accuracy of integrated PET-CT compared with dedicated PET alone for the staging of patients with nonsmall cell lung cancer. Ann Thorac Surg. 2004; 78:1017-1023.

9. Al-Sarraf N, Gately K, Lucey J, Wilson L, McGovern E, Young V. Lymph node staging by means of positron emission tomography is less accurate in non-small cell lung cancer patients with enlarged lymph nodes: Analysis of 1145 lymph nodes. Lung Cancer. 2008;60:62-68.

10. Roberts PF, Follette DM, von Haag D, et al. Factors associated with falsepositive staging of lung cancer by positron emission tomography. Ann Thorac Surg. 2000;70:1154-1159.

11. Freixinet Gilart J, García PG, de Castro FR, Suárez PR, Rodríguez NS, de Ugarte AV. Extended cervical mediastinoscopy in the staging of bronchogenic carcinoma. Ann Thorac Surg. 2000;70:1641-1643.

12. Inoue M, Nakagawa K, Fujiwara K, Fukuhara K, Yasumitsu T. Results of preoperative mediastinoscopy for small cell lung cancer. Ann Thorac Surg. 2000;70:1620-1623.

13. Hellwig D, Graeter TP, Ukena D, et al. ${ }^{18} \mathrm{~F}-\mathrm{FDG}$ PET for mediastinal staging of lung cancer: which SUV threshold makes sense? J Nucl Med. 2007;48:17611766.

14. Demura $\mathrm{Y}$, Tatsuro $\mathrm{T}$, Ishizaki $\mathrm{T}$, et al. ${ }^{18} \mathrm{~F}-\mathrm{FDG}$ accumulation with PET for differentiation between benign and malignant lesions in the thorax. J Nucl Med. 2003;44:540-548.

15. Gould MK, Maclean CC, Kuschner WG, Rydzak CE, Owens DK. Accuracy of positron emission tomography for diagnosis of pulmonary nodules and mass lesions: a meta-analysis. JAMA. 2001;285:914-924. 
16. Fischer BM, Mortensen J, Hojgaard L. Positron emission tomography in the diagnosis and staging of lung cancer: a systematic, quantitative review. Lancet Oncol. 2001;2:659-666.

17. El-Haddad G, Zhuang H, Gupta N, Alavi A. Evolving role of positron emission tomography in the management of patients with inflammatory and other benign disorders. Semin Nucl Med. 2004;34:313-329.

18. Goo JM, Im JG, Do KH, et al. Pulmonary tuberculoma evaluated by means of FDG PET: findings in 10 cases. Radiology. 2000;216:117-121.

19. Sazon DA, Santiago SM, Soo Hoo GW, et al. Fluorodeoxyglucose-positron emission tomography in the detection and staging of lung cancer. Am J Respir Crit Care Med. 1996;153:417-421.

20. Shim SS, Lee KS, Kim BT, et al. Non-small cell lung cancer: prospective comparison of integrated FDG PET/CT and CT alone for preoperative staging. Radiology. 2005;236:1011-1019.

21. Al-Sarraf N, Aziz R, Doddakula K, et al. Factors causing inaccurate staging of mediastinal nodal involvement in non-small cell lung cancer patients staged by positron emission tomography. Interact Cardiovasc Thorac Surg. 2007;6:350-353.

22. Ohno Y, Koyama H, Nogami M, et al. STIR turbo SE MR imaging vs. coregistered FDG-PET/CT: quantitative and qualitative assessment of N-stage in non-small-cell lung cancer patients. J Magn Reson Imaging. 2007;26:1071-1080.

23. Ohno Y, Hatabu H, Takenaka D, et al. Metastases in mediastinal and hilar lymph nodes in patients with non-small cell lung cancer: quantitative and qualitative assessment with STIR turbo spin-echo MR imaging. Radiology. 2004;231:872-879.

24. Takenaka D, Ohno Y, Hatabu H, et al. Differentiation of metastatic versus nonmetastatic mediastinal lymph nodes in patients with non-small cell lung cancer using respiratory-triggered short inversion time inversion recovery (STIR) turbo spin-echo MR imaging. Eur J Radiol. 2002;44:216-224.

25. Tournoy KG, Maddens S, Gosselin R, Van Maele G, van Meerbeeck JP, Kelles A. Integrated FDG-PET/CT does not make invasive staging of the intrathoracic lymph nodes in non-small cell lung cancer redundant: a prospective study. Thorax. 2007;62:696-701.

26. Delakis I, Moore EM, Leach MO, De Wilde JP. Developing a quality control protocol for diffusion imaging on a clinical MRI system. Phys Med Biol. 2004 21;49:1409-1422.
27. Svanholm H, Starklint H, Gundersen HJ, Fabricius J, Barlebo H, Olsen S. Reproducibility of histomorphologic diagnoses with special reference to the kappa statistic. APMIS. 1989;97:689-698.

28. Costabel U, Hunninghake GW. ATS/ERS/WASOG statement on sarcoidosis. Eur Respir J. 1999;14:735-737.

29. Swensen SJ, Brown LR, Colby TV, Weaver AL. Pulmonary nodules: CT evaluation of enhancement with iodinated contrast material. Radiology. 1995; 194:393-398.

30. Bottomley PA, Hardy CJ, Argersinger RE, Allen-Moore G. A review of ${ }^{1} \mathrm{H}$ nuclear magnetic resonance relaxation in pathology: are $\mathrm{T} 1$ and $\mathrm{T} 2$ diagnostic? Med Phys. 1987;14:1-37.

31. Yamashita Y, Yokoyama T, Tomiguchi S, Takahashi M, Ando M. MR imaging of focal lung lesions: elimination of flow and motion artifact by breath-hold ECGgated and black-blood techniques on T2-weighted turbo SE and STIR sequences. J Magn Reson Imaging. 1999;9:691-698.

32. Puderbach M, Hintze C, Ley S, Eichinger M, Kauczor HU, Biederer J. MR imaging of the chest: a practical approach at 1.5T. Eur J Radiol. 2007;64:345-355.

33. Abida KH. Sarcoidosis. In: Phillip TC, Timothy CA, Carlos B, eds. Color Atlas and Text of Pulmonary Pathology. Philadelphia, PA: Lippincott Williams \& Wilkins; 2005:259-260.

34. Halpern BS, Schiepers C, Weber WA, et al. Presurgical staging of non-small cell lung cancer: positron emission tomography, integrated positron emission tomography/CT, and software image fusion. Chest. 2005;128:2289-2297.

35. Fletcher JW, Djulbegovic B, Soares HP, et al. Recommendations on the use of ${ }^{18}$ F-FDG PET in oncology. J Nucl Med. 2008;49:480-508.

36. Yoshida Y, Kurokawa T, Sawamura Y, et al. Comparison of ${ }^{18}$ F-FDG PET and MRI in assessment of uterine smooth muscle tumors. J Nucl Med. 2008;49:708712 .

37. Catana C, Wu Y, Judenhofer MS, Qi J, Pichler BJ, Cherry SR. Simultaneous acquisition of multislice PET and MR images: initial results with a MRcompatible PET scanner. J Nucl Med. 2006;47:1968-1976.

38. Judenhofer MS, Catana C, Swann BK, et al. PET/MR images acquired with a compact MR-compatible PET detector in a 7-T magnet. Radiology. 2007;244: 807-814. 